University of New Orleans

ScholarWorks@UNO

2012

\title{
A note on acoustic propagation in power-law fluids: Compact kinks, mild discontinuities, and a connection to finite-scale theory
}

Dongming Wei

University of New Orleans, dwei@uno.edu

Follow this and additional works at: https://scholarworks.uno.edu/math_facpubs

Part of the Mathematics Commons

\section{Recommended Citation}

Wei, Dongming and Jordan; P.M. A note on acoustic propagation in power-law fluids: Compact kinks, mild discontinuities, and a connection to finite-scale theory. International Journal of Non-Linear Mechanics preprint

This Article is brought to you for free and open access by the Department of Mathematics at ScholarWorks@UNO. It has been accepted for inclusion in Mathematics Faculty Publications by an authorized administrator of ScholarWorks@UNO.For more information, please contact scholarworks@uno.edu. 


\title{
A note on acoustic propagation in power-law fluids: Compact kinks, mild discontinuities, and a connection to finite-scale theory
}

\author{
Dongming Wei ${ }^{\mathrm{a}}$, P.M. Jordan ${ }^{\mathrm{b}, *}$ \\ ${ }^{a}$ Department of Mathematics, University of New Orleans, New Orleans, LA 70148, USA \\ ${ }^{b}$ Acoustics Div., U.S. Naval Research Laboratory, Stennis Space Ctr., MS 39529, USA
}

\begin{abstract}
Acoustic traveling waves in a class of viscous, power-law fluids are investigated. Both bi-directional and unidirectional versions of the one-dimensional (1D), weakly-nonlinear equation of motion are derived; traveling wave solutions (TWS)s, special cases of which take the form of compact and algebraic kinks, are determined; and the impact of the bulk viscosity on the structure/nature of the kinks is examined. Most significantly, we point out a connection that exists between the power-law model considered here and the recently introduced theory of finite-scale equations.
\end{abstract}

Keywords: Nonlinear acoustics, power-law fluids, traveling wave solutions, finite-scale Navier-Stokes equations

\section{Introduction}

In what are commonly referred to as "power-law" fluids, the shear stress obeys the Ostwald-de Wael model [1, 2], at least over limited ranges of shear rate. Under this constitutive relation, the (constant) shear viscosity coefficient $\mu(>0)$ is replaced by the more general quantity

$$
\mu_{\mathrm{eff}}:=\mu k|\boldsymbol{\kappa}|^{n-1} \quad(k, n>0),
$$

where $\mu_{\mathrm{eff}}$ is called the effective shear viscosity [1]. Here, $\boldsymbol{\kappa}$ denotes the shear rate; $n$, the power-law index, and $k$, which is related to the consistency coefficient $\mathcal{K}$ of the Ostwald-de Wael model via $\mathcal{K}=\mu k$ [1], are empirically determined constants, where it should be noted that $k$ carries (SI) units of $\sec ^{n-1}$; and we observe that $k:=1$ when $n=1$, i.e., the Newtonian fluid case is recovered when $n=1$.

Physically, the cases $n \in(0,1)$ and $n>1$ correspond to fluids in which the viscosity decreases (shear-thinning) and increases (shear-thickening), respectively, with increasing $\boldsymbol{\kappa}$. Examples of the former, which are termed pseudoplastic, include polymer melts and polymer solutions; those of the latter, termed dilatant, include certain concentrated suspensions and other multiphase materials (see Ref. [1] and those therein).

While a great deal has been written regarding the application of the Ostwald-de Wael model to incompressible flows (again, see Ref. [1] and those therein), we are not aware of any body of work devoted to the study of acoustic phenomena in general power-law fluids. This, in spite of the fact that there are important practical and theoretical reasons to further our understanding of the nonlinear phenomena associated with the propagation of sound in nonNewtonian 11 fluids.

The present Note is put forth as a step towards filling this apparent "hole" in the acoustics literature; its aims are threefold: (i) derive in 1D the bi-directional, weakly-nonlinear, equation of motion for acoustic propagation in fluids whose effective shear viscosity is described by (1); (ii) integrate this PDE under the traveling wave assumption and analyze the resulting solutions, which fall into two classes, using analytical and numerical methods; and (iii), demonstrate connections that exist between a special case of (1) and both the "artificial viscosity" method of von Neumann and Richtmyer [4] and the compressible version of what has come to be known as finite-scale NavierStokes theory, a mathematically rigorous approach to turbulence modeling recently introduced by Margolin [5, 6].

${ }^{*}$ Corresponding author. Tel.: +1-228-688-4338; fax: +1-228-688-5049

Email address: pjordan@nrlssc.navy .mil (P.M. Jordan)

${ }^{1}$ It should be mentioned here that Straughan [3] has investigated nonlinear acoustic waves in inviscid dipolar fluids. 


\section{Mathematical formulation}

\subsection{Governing equations and constitutive assumptions}

We begin by listing the equations governing the flow a viscous, compressible fluid in which the effects of viscous shear are described by (1). Confining our attention to planar flow perpendicular to and along the $x$-axis, the velocity and heat flux vectors assume the form $\mathbf{u}=(u(x, t), 0,0)$ and $\mathbf{q}=(q(x, t), 0,0)$, respectively, while the mass density $\varrho$, thermodynamic pressure $\wp$, absolute temperature $\vartheta$, and specific entropy $\eta$ become functions of $x$ and $t$ only. Thus, conservation of mass dictates that

$$
\varrho_{t}+(\varrho u)_{x}=0
$$

the momentum equation 2 takes the form

$$
\varrho\left(u_{t}+u u_{x}\right)=-\wp_{x}+\partial_{x}\left[\left(\mu_{\mathrm{B}}+\frac{4}{3} \mu k\left|u_{x}\right|^{n-1}\right) u_{x}\right]
$$

from which the absence of all body forces has been assumed; and the linearized energy equation, an approximation of great importance in weakly-nonlinear fluid-acoustics, is [7, p. 21]

$$
\varrho_{\mathrm{e}} \vartheta_{\mathrm{e}} \eta_{t}=K \vartheta_{x x}
$$

Here, $\mathbf{q}$ is assumed to satisfy Fourier's law; $\mu_{\mathrm{B}}(\geq 0)$ is the bulk viscosity and $K(>0)$ is the thermal conductivity, both of which we take to be constant; the notation $\partial_{\varsigma}:=\partial / \partial \varsigma$ is employed for convenience; and we note for future reference that $u=\phi_{x}$, where $\phi=\phi(x, t)$ is the velocity potential, since the irrotationality condition $\nabla \times \mathbf{u}=0$ is identically satisfied under the assumed flow geometry.

To close this system, a (constitutive) relation between the thermodynamic variables present is required. In the present investigation, we assume the usual quadratic approximation to the general, non-isentropic equation of state $\wp=\wp(\varrho, \eta)$, namely,

$$
\wp=\wp_{\mathrm{e}}+\varrho_{\mathrm{e}} c_{\mathrm{e}}^{2}\left[s+(\beta-1) s^{2}+\left(\frac{\gamma-1}{\chi c_{\mathrm{e}}^{2}}\right)\left(\eta-\eta_{\mathrm{e}}\right)\right]
$$

which is valid for both gases and liquids provided fluctuations in $\varrho$ and $\eta$ about their equilibrium state values are sufficiently small; see Ref. [8, 9]. Here, $s=\left(\varrho-\varrho_{\mathrm{e}}\right) / \varrho_{\mathrm{e}}$ is termed the condensation; $\beta(>1)$, a constant, is known as the coefficient of nonlinearity [9]; $\gamma(>1)$, the adiabatic index [10], is defined as $\gamma=c_{p} / c_{\nu}$, where the constants $c_{p}>c_{v}>0$ respectively denote the specific heats at constant pressure and volume; $c_{\mathrm{e}}(>0)$, the adiabatic sound speed, is also a constant and represents the speed of sound in the undisturbed fluid; $\chi(>0)$ is the thermal coefficient of volume expansion; and we assume that the equilibrium state values of all quantities, which are those appended by an "e" subscript, are constants.

In what follows, we shall ignore the effects of thermal conduction; i.e., in place of (4) we make the (further) approximation $\eta_{t}=0$. If we integrate this most specialized (and simple) case of the energy equation subject to the initial condition $\eta(x, 0)=\eta_{\mathrm{e}}$, then it is trivially established that

$$
\eta(x, t)=\eta_{\mathrm{e}}
$$

Hence, we see that neglecting the RHS of (4) has, in the present setting, caused the flow to become homentropic [10].

Remark 1. In the case of a perfect gas $[10], c_{\mathrm{e}}=\sqrt{\gamma \wp_{\mathrm{e}} / \varrho_{\mathrm{e}}}$, the coefficient of the last (i.e., entropy) term in (5) reduces to $c_{p}^{-1}[11]$, and the coefficient of nonlinearity can be expressed in terms of the ratio of specific heats via the simple relation $\beta=(\gamma+1) / 2$; see [9].

\footnotetext{
${ }^{2}$ Of course, 3 is the momentum equation for only the $x$-component of $\mathbf{u}$; in contrast, those for the $y$ - and $z$-components have become simply $\wp_{y}=0$ and $\wp_{z}=0$, respectively, under the assumed flow geometry.
} 


\subsection{Deriving a bi-directional, weakly-nonlinear equation of motion}

To this end, we first substitute (6) into (5), thus eliminating $\eta$ from further consideration and reducing the latter to

$$
\wp=\wp_{\mathrm{e}}+\varrho_{\mathrm{e}} c_{\mathrm{e}}^{2}\left[s+(\beta-1) s^{2}\right] \quad(-1 \ll s \ll 1) .
$$

In turn, using (7) to eliminate $\wp$ from (3) yields

$$
\varrho\left(u_{t}+u u_{x}\right)=-\varrho_{\mathrm{e}} c_{\mathrm{e}}^{2}\left[s+(\beta-1) s^{2}\right]_{x}+\mu \partial_{x}\left[\left(\mu_{\mathrm{B}} / \mu+\frac{4}{3} k\left|u_{x}\right|^{n-1}\right) u_{x}\right] .
$$

Next, we introduce the following dimensionless quantities:

$$
\phi^{\diamond}=\phi /(V \mathrm{~L}), \quad u^{\diamond}=u / V, \quad x^{\diamond}=x / \mathrm{L}, \quad t^{\diamond}=t\left(c_{\mathrm{e}} / \mathrm{L}\right)
$$

where the positive constants $V$ and $L$ denote a characteristic speed and (macroscopic) length, respectively, and replace $\varrho$ with $\varrho_{\mathrm{e}}(1+s)$ in (2) and (8). Thus, after a few additional manipulations, the former and latter equations become

$$
s_{t}+\epsilon s_{x} \phi_{x}+\epsilon \phi_{x x}(1+s)=0
$$

$$
\epsilon(1+s) \partial_{x}\left[\phi_{t}+\frac{1}{2} \epsilon\left(\phi_{x}\right)^{2}\right]=-\partial_{x}\left[s+(\beta-1) s^{2}\right]+\epsilon(\operatorname{Re})^{-1} \partial_{x}\left[\left(\mu_{\mathrm{B}} / \mu+\frac{4}{3} \sigma\left|\phi_{x x}\right|^{n-1}\right) \phi_{x x}\right] .
$$

In this system, $\epsilon=V / c_{\mathrm{e}}$ is the Mach number, where the weakly-nonlinear approximation requires that $\epsilon \ll 1$ be assumed henceforth; $\operatorname{Re}=c_{\mathrm{e}} \mathrm{L} / v$ is a Reynolds number, where $v=\mu / \varrho_{\mathrm{e}}$ denotes the kinematic viscosity; we have set $\sigma:=k(V / \mathrm{L})^{n-1}$, where we note that $\sigma=1$ when $n=1$; and here and henceforth, all diamond superscripts are suppressed for typographical convenience.

Dividing (11) by $\epsilon(1+s)$ and then expanding in a binomial series under the assumption $|s|=O(\epsilon)$, another demand of the weakly-nonlinear approximation, yields, after re-arranging terms and simplifying,

$$
\partial_{x}\left\{\phi_{t}+\frac{1}{2} \epsilon\left(\phi_{x}\right)^{2}+\epsilon^{-1}\left[s+(\beta-3 / 2) s^{2}+\cdots\right]\right\}=(\operatorname{Re})^{-1}(1-s+\cdots) \partial_{x}\left[\left(\mu_{\mathrm{B}} / \mu+\frac{4}{3} \sigma\left|\phi_{x x}\right|^{n-1}\right) \phi_{x x}\right] .
$$

Neglecting terms of $O\left(\epsilon^{2}\right)$ and $O(\epsilon / \mathrm{Re})$ and then applying $\partial_{t}$ to both sides of Eq. (12) yields, after some rearrangement of term,

$$
\partial_{x}\left\{\phi_{t t}+\frac{1}{2} \epsilon \partial_{t}\left(\phi_{x}\right)^{2}+\epsilon^{-1}[1+2(\beta-3 / 2) s] s_{t}-(\operatorname{Re})^{-1} \partial_{t}\left[\left(\mu_{\mathrm{B}} / \mu+\frac{4}{3} \sigma\left|\phi_{x x}\right|^{n-1}\right) \phi_{x x}\right]\right\}=0,
$$

from which it follows that

$$
\phi_{t t}+\frac{1}{2} \epsilon \partial_{t}\left(\phi_{x}\right)^{2}+\epsilon^{-1}[1+2(\beta-3 / 2) s] s_{t}=(\operatorname{Re})^{-1} \partial_{t}\left[\left(\mu_{\mathrm{B}} / \mu+\frac{4}{3} \sigma\left|\phi_{x x}\right|^{n-1}\right) \phi_{x x}\right],
$$

where the resulting function of integration has been set to zero.

Finally, if we now eliminate $s_{t}$ in (14) using (10), followed by the elimination of $s$ and $s_{x}$ in the resulting expression using the relation $s=-\epsilon \phi_{t}+O\left(\epsilon^{2}\right)$, then, after neglecting terms of $O\left(\epsilon^{2}\right)$ and simplifying, we obtain a single, weaklynonlinear equation of motion in terms of the velocity potential, specifically,

$$
\phi_{t t}-\left[1-2 \epsilon(\beta-1) \phi_{t}\right] \phi_{x x}+\epsilon \partial_{t}\left(\phi_{x}\right)^{2}=(\operatorname{Re})^{-1} \partial_{t}\left[\left(\mu_{\mathrm{B}} / \mu+\frac{4}{3} \sigma\left|\phi_{x x}\right|^{n-1}\right) \phi_{x x}\right] .
$$

Here, it should be noted that, had we not neglected the RHS of 44), then the (positive) quantity $(\gamma-1) / \operatorname{Pr}$ would have been added to the ratio $\mu_{\mathrm{B}} / \mu$ in [15], where $\operatorname{Pr}=c_{p} \mu / K$ denotes the Prandtl number [2, p. 80].

At this juncture, the cases $\mu_{\mathrm{B}}=0$, which corresponds to monatomic gases, and $\mu_{\mathrm{B}}>0$, which corresponds to most/all other common fluids under ordinary conditions [10], must be treated separately, an action made necessary by the degeneracy created (in (15) when $\mu_{\mathrm{B}}=0$ is taken.

Remark 3. In the case of a Newtonian fluid, i.e., for $n=1$, 15 reduces to

$$
\phi_{t t}-\left[1-2 \epsilon(\beta-1) \phi_{t}\right] \phi_{x x}+\epsilon \partial_{t}\left(\phi_{x}\right)^{2}=\left(\operatorname{Re}_{\ell}\right)^{-1} \phi_{t x x},
$$

which is the special case of the Blackstock-Lesser-Seebass-Crighton (BLSC) equation corresponding to a nonthermally conducting Newtonian fluid; see, e.g., Ref. [12] and those therein. Here, $\operatorname{Re}_{\ell}=c_{\mathrm{e}} \mathrm{L} / v_{\ell}$ is a second Reynolds number, where $v_{\ell}:=v\left(\frac{4}{3}+v_{\mathrm{B}} / v\right)$ can be termed the kinematic longitudinal coefficient of viscosity [13, p. 38] and we have set $v_{\mathrm{B}}:=\varrho_{\mathrm{e}}^{-1} \mu_{\mathrm{B}}$. 


\section{Traveling waves: Monatomic gases}

\subsection{Ansatz and associated ODE}

Considering the case $\mu_{\mathrm{B}}=0$ first, we begin our analysis with the following observation: Since (15) is invariant under the transformation $x \mapsto-x$, we need only seek, without loss of generality, right-running waveforms. Introducing then the ansatz $\phi(x, t)=F(\xi)$, where $\xi=x-\lambda t$ is the wave variable and the (constant) wave speed $\lambda$ is strictly positive, we integrate once wrt $\xi$; as a result, (15) reduces to the ODE

$$
\lambda v^{*} f^{\prime}\left|f^{\prime}\right|^{n-1}=\left(1-\lambda^{2}\right) f+\epsilon \beta \lambda f^{2}+\mathfrak{K}_{1}, \quad \text { where } \quad v^{*}:=\frac{4}{3}\left(\frac{\sigma}{\operatorname{Re}}\right),
$$

a prime denotes $\mathrm{d} / \mathrm{d} \xi$, we have set $f(\xi):=F^{\prime}(\xi)$, and $\mathfrak{K}_{1}$ is the constant of integration. Assuming TWSs in the form of kinks [14], we impose and enforce the asymptotic conditions $f \rightarrow 1,0$ as $\xi \rightarrow \mp \infty$. Thus, $\mathfrak{K}_{1}=0$ and, just as in the case of (16), the speed of the (dispersed) shock-front is

$$
\lambda=\frac{\epsilon \beta+\sqrt{4+\epsilon^{2} \beta^{2}}}{2},
$$

where we observe that $\lambda>1$; see Ref. [12, §3.1]. Solving now for $f^{\prime}$, we obtain the associated ODE for the case of monatomic gases, namely,

$$
-f^{\prime}=\varkappa\left(f-f^{2}\right)^{1 / n}=\varkappa \Lambda(f), \quad \text { where } \quad \varkappa:=\left(\frac{\epsilon \beta}{\nu^{*}}\right)^{1 / n} .
$$

Here, $\left|f^{\prime}\right|$ has been replaced with $-f^{\prime}$ since $f^{\prime} \leq 0$ is expected in the case of right-running kinks.

\subsection{Stability results and quadrature}

Clearly, the equilibria of (19) are $\bar{f}=\{0,1\}$. It is also clear that $\Lambda(f) \in C^{1}[0,1]$ when $n<1$. For $n>1$, however, $\Lambda(f) \in C[0,1]$ but, since

$$
\lim _{f \rightarrow 0^{+}}\left|\frac{\mathrm{d} \Lambda}{\mathrm{d} f}\right|=\lim _{f \rightarrow 1^{-}}\left(\frac{\mathrm{d} \Lambda}{\mathrm{d} f}\right)=\infty \quad(n>1), \quad \text { where } \quad \frac{\mathrm{d} \Lambda}{\mathrm{d} f}=\frac{1-2 f}{n\left(f-f^{2}\right)^{1-1 / n}},
$$

$\Lambda(f) \notin C^{1}[0,1]$. Thus, when $n>1$ the slope of the phase portrait, i.e., the plot of $\Lambda$ vs. $f$, is undefined at both equilibria. This means that at neither equilibria is the Lipschitz condition satisfied; therefore, uniquenes 3 is not assured at either equilibria when $n>1$.

Returning now to 19), we let $m:=n^{-1}$ (to simplify the typesetting), separate variables, and then integrate, the result of which is the quadrature

$$
\int \frac{\mathrm{d} f}{\left(f-f^{2}\right)^{m}}=-\varkappa \xi+\mathfrak{K}_{2} \quad(0<f<1),
$$

where $\mathfrak{K}_{2}$ is the constant of integration. Because the analytical structure of the resulting integral curves differs, the cases $m \geq 1$ and $m<1$ will be considered separately. Moreover, for both convenience of presentation and, more importantly, ensuring the TWSs obtained are bounded over the entire real line, in what follows, $f(0)=1 / 2$ (i.e., $f=1 / 2$ at the wavefront $\xi=0$ ) shall, without loss of generality, henceforth be assumed.

\footnotetext{
${ }^{3}$ For an interesting discussion of this issue in the context of nonlinear elasticity, see Saccomandi [15].
} 


\subsection{The case $m \geq 1$}

Expanding the integrand above in a binomial series, which is permissible in this case since $f(0) \in(0,1)$ implies that the resulting primitive will be such that $f \in(0,1)$, and then integrating term-by-term, we obtain, after solving for $\mathfrak{K}_{2}$ and simplifying, the following exact (but generally implicit) solution:

$$
\varkappa \xi=-\sum_{k=0}^{\infty} T_{k}(f), \quad \text { for } \quad 0<f<1 .
$$

Here,

$$
T_{k}(f)= \begin{cases}\frac{\Gamma(m+k)\left[f^{k-m+1}-\left(\frac{1}{2}\right)^{k-m+1}\right]}{\Gamma(m)(k-m+1) k !}, & k \neq m-1, \\ \frac{\Gamma(2 m-1) \ln (2 f)}{\Gamma^{2}(m)}, & k=m-1,\end{cases}
$$

where $\Gamma(\cdot)$ denotes the gamma function.

Remark 4. For $m=1$, the series in (22) can be summed exactly; the result is, after simplifying,

$$
-\varkappa \xi=\ln (2 f)+\sum_{k=1}^{\infty} \frac{f^{k}-\left(\frac{1}{2}\right)^{k}}{k}=\ln \left(\frac{f}{1-f}\right),
$$

from which it is easily established that $f$ in this case assumes the form of a Taylor shock, i.e.,

$$
f(\xi)=\frac{\exp (-\varkappa \xi)}{1+\exp (-\varkappa \xi)}=\frac{1}{2}\left[1-\tanh \left(\frac{1}{2} \varkappa \xi\right)\right] \quad(m=1) .
$$

Thus, as expected, setting $m=(n=) 1$ allows us to recover the well known TWS of Burgers' equation.

Remark 5. When $m=3 / 2$, the series in (22) can again be summed exactly and the following explicit solution easily established:

$$
f(\xi)=\frac{16+\varkappa^{2} \xi^{2}-\varkappa \xi \sqrt{16+\varkappa^{2} \xi^{2}}}{2\left(16+\varkappa^{2} \xi^{2}\right)} \quad(m=3 / 2),
$$

from which we see that $f$ in this case assumes the form of an algebraic kink.

\subsection{The case $m<1$}

In this case the integral in (21) can be directly evaluated, and closed-form expressions obtained. However, care must now be exercised because when $m<1, \bar{f}=\{0,1\}$ are no longer the asymptotic limiting values of $f$; instead, they are the equations which define the envelope 4 [16] of the one-parameter (i.e., $\mathfrak{K}_{2}$ ) family of integral curves that satisfy (19). What this means, of course, is the following: If $m<1$, then $f(\xi)=0,1$ are attained at finite values of $\xi$.

Using the Integrate [] command, which is part of the software package Mathematica (ver. 5.2), it is a straightforward matter to establish that if $m<1$, then the following satisfies both (19) and the wavefront condition $f(0)=1 / 2$ :

$$
f(\xi)=\left\{\begin{array}{ll}
1, & \text { for } \xi \leq \xi_{\mathrm{L}} ; \\
0, & \text { for } \xi \geq \xi_{\mathrm{R}} ;
\end{array} \quad \varkappa \xi=\frac{{ }_{2} F_{1}(1-m, m ; 2-m ; f)}{(m-1) f^{m-1}}-\frac{4^{m-1} \Gamma(2-m) \sqrt{\pi}}{(m-1) \Gamma(3 / 2-m)}, \quad \text { for } \quad 0<f<1 .\right.
$$

Here, ${ }_{2} F_{1}$ denotes the Gauss hypergeometric series,

$$
\xi_{\mathrm{L}}=\frac{-\Gamma(2-m) \sqrt{\pi}}{4^{1-m} \varkappa(1-m) \Gamma(3 / 2-m)}, \quad \text { and } \quad \xi_{\mathrm{R}}=\frac{\left[m^{2}(9-m)+2(12-13 m)\right] \Gamma(2-m) \sqrt{\pi}}{4^{1-m} \varkappa(1-m)(2-m)(3-m)(4-m) \Gamma(3 / 2-m)} .
$$

Remark 6. For $m=1 / 2$, 27] yields the explicit solution

$$
f(\xi)=\left\{\begin{array}{ll}
1, & \xi \leq-\frac{1}{2} \pi \varkappa^{-1} \\
\sin ^{2}\left[\frac{1}{2}\left(\varkappa \xi-\frac{1}{2} \pi\right)\right], & -\frac{1}{2} \pi \varkappa^{-1}<\xi<\frac{1}{2} \pi \varkappa^{-1} \\
0, & \xi \geq \frac{1}{2} \pi \varkappa^{-1}
\end{array} \quad(m=1 / 2),\right.
$$

a result which can also be obtained from Ref. [4, p. 234]. Here, $\xi_{\mathrm{L}, \mathrm{R}}$ reduce to $\mp \frac{1}{2} \pi \varkappa^{-1}$, respectively.

${ }^{4}$ Recall the discussion in sect. 3.2 regarding the question of uniqueness. 


\subsection{Mild discontinuities}

For the case $m<1$, it can be shown that

$$
\llbracket f^{\prime \prime} \rrbracket_{\mathrm{L}}=\llbracket f^{\prime \prime} \rrbracket_{\mathrm{R}}=\left\{\begin{array}{ll}
\infty, & m \in\left(0, \frac{1}{2}\right) \\
\frac{1}{2} \varkappa^{2}, & m=\frac{1}{2} \\
0, & m \in\left(\frac{1}{2}, 1\right)
\end{array} \quad\left(\mu_{\mathrm{B}}=0\right),\right.
$$

where $\llbracket \mathfrak{F} \rrbracket_{\mathrm{L}, \mathrm{R}}$, the amplitudes of the jumps suffered by a function $\mathfrak{F}$ across the planes $\xi=\xi_{\mathrm{L}, \mathrm{R}}$, are defined here as

$$
\llbracket \mathfrak{F} \rrbracket_{\mathrm{L}}:=\lim _{\xi \rightarrow \xi_{\mathrm{L}}^{-}} \mathfrak{F}-\lim _{\xi \rightarrow \xi_{\mathrm{L}}^{+}} \mathfrak{F} \quad \text { and } \quad \llbracket \mathfrak{F} \rrbracket_{\mathrm{R}}:=\lim _{\xi \rightarrow \xi_{\mathrm{R}}^{-}} \mathfrak{F}-\lim _{\xi \rightarrow \xi_{\mathrm{R}}^{+}} \mathfrak{F}
$$

and we have used the fact that

$$
\begin{aligned}
f^{\prime \prime} & =-\varkappa^{2}(1-m)^{2}\left\{\frac{\mathrm{d}}{\mathrm{d} f}\left[\frac{{ }_{2} F_{1}(1-m, m ; 2-m ; f)}{f^{m-1}}\right]\right\}^{-3} \frac{\mathrm{d}^{2}}{\mathrm{~d} f^{2}}\left[\frac{{ }_{2} F_{1}(1-m, m ; 2-m ; f)}{f^{m-1}}\right] \\
& =m \varkappa^{2}(1-2 f)\left(f-f^{2}\right)^{2 m-1} .
\end{aligned}
$$

Evidently, if $0<m \leq \frac{1}{2}$, then $f \in C^{1}(\mathbb{R})$ but $f \notin C^{2}(\mathbb{R})$, with the case $m=1 / 2$ (i.e., $n=2$ ) admitting mild discontinuities of the lowest possible order, namely, two; see Ref. [17]. In contrast, $f \in C^{2}(\mathbb{R})$ for every $\frac{1}{2}<m<1$; however, it can be shown that higher-order mild discontinuities do occur for certain values of $m$ in this (upper) range: If $m=1-1 / N$, where $N \geq 3$ is an integer, then $\left|\llbracket f^{(N)} \rrbracket_{\mathrm{L}, \mathrm{R}}\right| \propto \varkappa^{N}$.

Remark 7. For $m \in\left(0, \frac{1}{2}\right)$, the compact kinks described in this section are, qualitatively, very similar to those uncovered by Destrade et al. [18], who considered transverse traveling waves in a class of nonlinear viscoelastic media.

Remark 8. It is noteworthy that acoustic mild discontinuities of order two have also been predicted in inviscid dipolar fluids; again, see Ref. [3, §3], wherein such discontinuities are termed "dipolar stress waves".

\section{Traveling waves: Liquids and non-monatomic gases}

\subsection{Ansatz, associated ODEs, and representative special cases}

Seeking TWSs as we did in sect. 3.1, but now under the assumption $\mu_{\mathrm{B}}>0$, it is readily established that the associated ODE in this case is

$$
\alpha\left(-f^{\prime}\right)^{n}-f^{\prime}=\varkappa^{\bullet}\left(f-f^{2}\right)
$$

where we have set

$$
\alpha:=\frac{4}{3}\left(\frac{\sigma \mu}{\mu_{\mathrm{B}}}\right) \quad \text { and } \quad \varkappa^{\bullet}:=\operatorname{Re}\left(\frac{\epsilon \beta \mu}{\mu_{\mathrm{B}}}\right) .
$$

Here, once again, we have assumed the ansatz $\phi(x, t)=F(\xi)$, with $f=F^{\prime}$; the wave speed is given by (18); and we have imposed and enforced the asymptotic conditions $f \rightarrow 1,0$ as $\xi \rightarrow \mp \infty$.

If $n=1, \frac{1}{2}, 2,3,4$, then one can solve for $f^{\prime}$ in terms of $f$; indeed, with little difficulty it can be shown that

$$
f^{\prime}=-\frac{1}{2} \begin{cases}2 \varkappa^{\bullet}(1+\alpha)^{-1}\left(f-f^{2}\right), & n=1, \\ \alpha^{2}+2 \varkappa^{\bullet} f(1-f)-\alpha \sqrt{\alpha^{2}+4 \varkappa^{\bullet} f(1-f)}, & n=\frac{1}{2}, \\ -\alpha^{-1}\left[1-\sqrt{1+4 \alpha \varkappa^{\bullet} f(1-f)}\right], & n=2,\end{cases}
$$

while obtaining such expressions for the cases $n=3,4$ requires use of the more complicated formulas of Cardano and Ferrari, respectively. Additionally, phase-plane analyses reveal that all three ODEs in (35) admit kink-type TWSs, provided of course that $f(0) \in(0,1)$, where we recall that the $n=1$ case corresponds to Burgers' equation. 
where we have again taken $f(0)=1 / 2$ and

$$
\mathcal{F}(\mathfrak{f}):=-\frac{1}{\varkappa^{\bullet}}\left[(-\mathfrak{f})+\alpha(-\mathfrak{f})^{n}\right] \quad(\mathfrak{f}<0) .
$$

where in the present context $l_{n}:=\left|f^{\prime}(0)\right|^{-1}$ for all $n>0$.

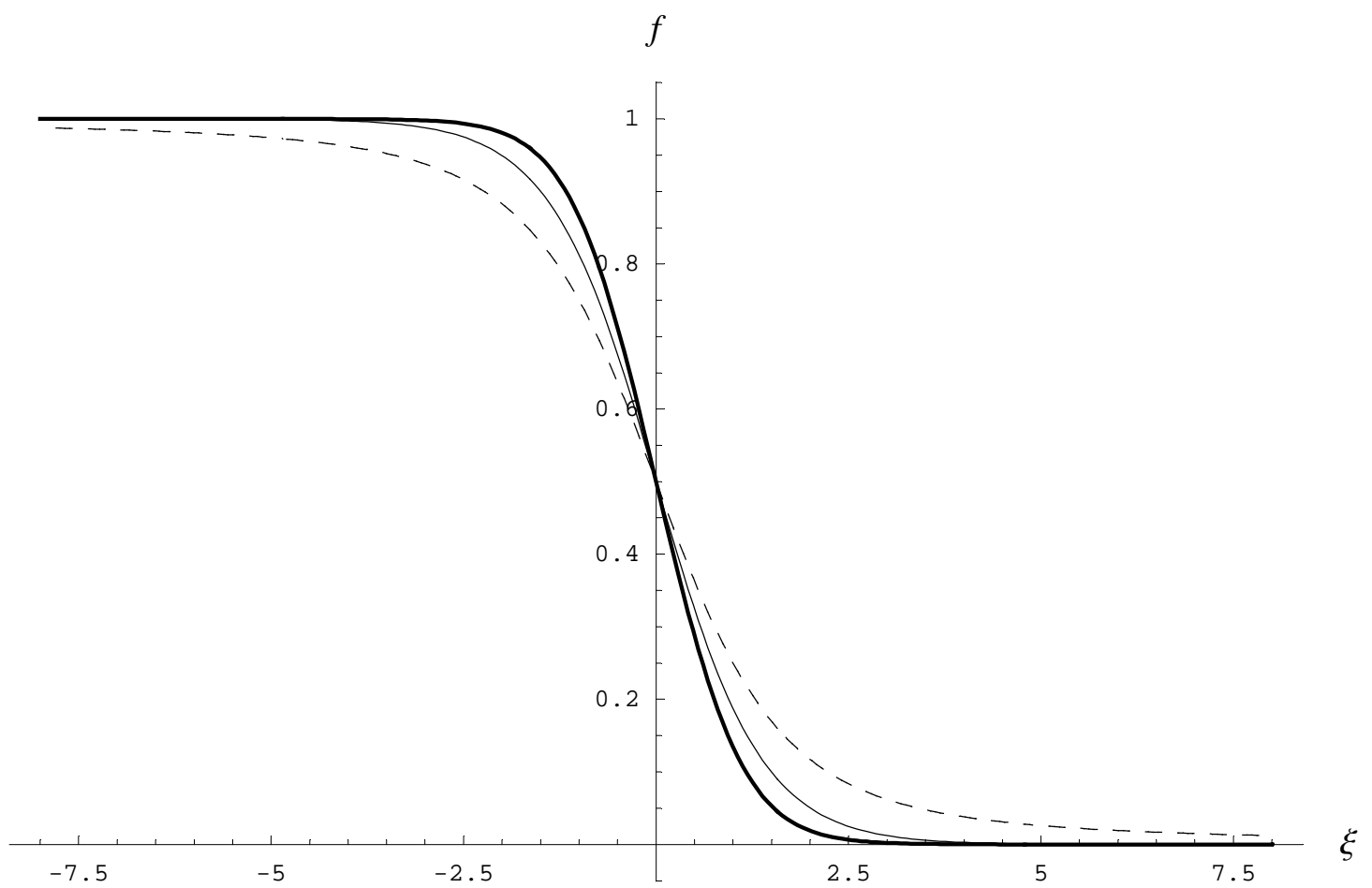

Figure 1: $f$ vs. $\xi$ based on 33 for $\alpha=0.5$ and $\varkappa^{\bullet}=2.2$. Bold-solid: $n=2$. Thin-solid: $n=1$ (Burgers' equation). Thin-broken: $n=1 / 2$. 


\subsection{The special case $\mu_{\mathrm{B}}>0, n=2$}

In this subsection we present a number of analytical results, including the exact solution, for this important special case; one which will figure prominently in sect. 6 below. Our purpose here is to illustrate, to a limited extent at least, the behavior of the resulting kink profile, as well as examine the case of vanishing $\mu_{\mathrm{B}}$.

Now, on integrating the $n=2$ case of (35) and then enforcing the wavefront condition $f(0)=1 / 2$, the following exact, but implicit, expression for the velocity field is obtained:

$$
\begin{aligned}
-\xi=\frac{1}{\varkappa^{\bullet}}\left\{\sqrt{\alpha \varkappa^{\bullet}} \sin ^{-1}\left[\frac{2 \sqrt{\alpha \varkappa^{\bullet}}\left(f-\frac{1}{2}\right)}{\sqrt{1+\alpha \varkappa^{\bullet}}}\right]+\tanh ^{-1}[2(f\right. & \left.\left.-\frac{1}{2}\right)\right] \\
& \left.+\tanh ^{-1}\left[\frac{2\left(f-\frac{1}{2}\right)}{\sqrt{1+\alpha \varkappa^{\bullet}-4 \alpha \varkappa^{\bullet}\left(f-\frac{1}{2}\right)^{2}}}\right]\right\}, \quad f \in(0,1) .
\end{aligned}
$$

While exact, the solution we have just determined is also quite complicated, and as such provides little in the way of physical insight. Fortunately, however, approximate/asymptotic expression for $f$, which are both simpler than their exact counterpart and explicit, can be derived directly from (39).

To begin with, we expand the RHS of (39) about $f=1 / 2$. This yields, after simplifying, the power series

$$
-\xi=\frac{2}{\varkappa^{\bullet}}\left\{\left(1+\sqrt{1+\alpha \varkappa^{\bullet}}\right)\left(f-\frac{1}{2}\right)+\frac{2}{3}\left(\frac{2+\alpha \varkappa^{\bullet}+2 \sqrt{1+\alpha \varkappa^{\bullet}}}{\sqrt{1+\alpha \varkappa^{\bullet}}}\right)\left(f-\frac{1}{2}\right)^{3}+O\left[\left(f-\frac{1}{2}\right)^{4}\right]\right\}, \quad\left|f-\frac{1}{2}\right|<1,
$$

from which an explicit small-|$|\xi|$ approximation for $f$ can be obtained by neglecting terms of $O\left[(f-1 / 2)^{4}\right]$ and then using Cardano's formula to solve the resulting cubic.

In contrast, expanding the RHS of (39) about $f=0$ and then neglecting terms of $O\left(f^{2}\right)$, it is not difficult to derive the following explicit large- $\xi$ approximation:

$$
f(\xi) \approx\left(1+\alpha \varkappa^{\bullet}\right)^{-1} W_{0}\left\{\sqrt{1+\alpha \varkappa^{\bullet}} \exp \left[\sqrt{\alpha \varkappa^{\bullet}} \sin ^{-1}\left(\sqrt{\frac{\alpha \varkappa^{\bullet}}{1+\alpha \varkappa^{\bullet}}}\right)\right] \exp \left(-\varkappa^{\bullet} \xi\right)\right\} \quad\left(\xi \gg 1 / \varkappa^{\bullet}\right),
$$

from which it is a simple matter to establish that under the present special case,

$$
f(\xi) \sim\left(1+\alpha \varkappa^{\bullet}\right)^{-1 / 2} \exp \left[\sqrt{\alpha \varkappa^{\bullet}} \sin ^{-1}\left(\sqrt{\frac{\alpha \varkappa^{\bullet}}{1+\alpha \varkappa^{\bullet}}}\right)\right] \exp \left(-\varkappa^{\bullet} \xi\right) \quad(\xi \rightarrow \infty) .
$$

Here, $W_{0}(\cdot)$ denotes the principal branch of the Lambert $W$-function; see, e.g., Ref. [19, Appendix B] and those therein. In the interest of brevity, we leave the task of determining the corresponding expressions for the $\xi=-\infty$ asymptotic limit of $f$ to the reader.

In concluding this subsection we observe that 39 ) admits the small $-\mu_{\mathrm{B}}$ approximation

$$
f(\zeta) \approx \begin{cases}1, & \zeta \leq-\zeta_{\mathrm{c}} \\ \sin ^{2}\left[\frac{1}{2}\left(\sqrt{b} \zeta-\frac{1}{2} \pi\right)\right], & -\zeta_{\mathrm{c}}<\zeta<\zeta_{\mathrm{c}} \\ 0, & \zeta \geq \zeta_{\mathrm{c}}\end{cases}
$$

where, for convenience, we have set $\zeta:=\xi /(2 \alpha), b:=4 \alpha \varkappa^{\circ}$, and $\zeta_{c}=\frac{1}{2} \pi b^{-1 / 2}$. As expected, we find that (39) assumes the character of (29) when $\mu_{\mathrm{B}}$ is "near" zero.

\section{Unidirectional approximation: A generalized Burgers' equation for propagation in power-law fluids}

First, we divide (15) by $\left[1-2 \epsilon(\beta-1) \phi_{t}\right]$, which can never be zero, expand each occurrence of the reciprocal of this quantity in a binomial series (since $\epsilon \ll 1$ ), and then neglect terms $O\left(\epsilon^{2}\right)$ and $O(\epsilon / \mathrm{Re}$ ). Thus, after rearranging terms and simplifying, our equation of motion becomes

$$
\phi_{t t}-\phi_{x x}+\epsilon \partial_{t}\left[\left(\phi_{x}\right)^{2}+(\beta-1)\left(\phi_{t}\right)^{2}\right]=(\operatorname{Re})^{-1} \partial_{t}\left[\left(\mu_{\mathrm{B}} / \mu+\frac{4}{3} \sigma\left|\phi_{x x}\right|^{n-1}\right) \phi_{x x}\right],
$$


which for $n=1$ becomes the special case of Kuznetsov's equation [11, 12] corresponding to a non-thermally conducting Newtonian fluid.

Since we have confined our attention to right-running waves, we can, based on the $O(1)$ approximation $\phi_{x} \simeq-\phi_{t}$, replace5 the wave operator and the nonlinear (i.e., "small") term $\left(\phi_{t}\right)^{2}$ on the LHS of (44) with $2 \partial_{t}\left(\partial_{t}+\partial_{x}\right)$ and $\left(\phi_{x}\right)^{2}$, respectively. This then leads us to consider

$$
2 \partial_{t}\left(\partial_{t}+\partial_{x}\right) \phi+\epsilon \beta \partial_{t}\left(\phi_{x}\right)^{2}=(\operatorname{Re})^{-1} \partial_{t}\left[\left(\mu_{\mathrm{B}} / \mu+\frac{4}{3} \sigma\left|\phi_{x x}\right|^{n-1}\right) \phi_{x x}\right],
$$

which after integrating with respect to $t$ and then differentiating with respect to $x$ becomes

$$
2\left(\partial_{t}+\partial_{x}\right) u+2 \epsilon \beta u u_{x}=(\operatorname{Re})^{-1} \partial_{x}\left[\left(\mu_{\mathrm{B}} / \mu+\frac{4}{3} \sigma\left|u_{x}\right|^{n-1}\right) u_{x}\right],
$$

where we have used the fact that $u=\phi_{x}$. Introducing the change of variables $\mathrm{x}=x-t$ and $\mathrm{t}=t$, and then dividing by two, (46), reduces to

$$
u_{\mathrm{t}}+\epsilon \beta u u_{\mathrm{x}}=\frac{1}{2}(\operatorname{Re})^{-1} \partial_{\mathrm{x}}\left[\left(\mu_{\mathrm{B}} / \mu+\frac{4}{3} \sigma\left|u_{\mathrm{x}}\right|^{n-1}\right) u_{\mathrm{x}}\right] .
$$

Here, we observe that for $n=1$, [47) reduces to the classic Burgers' equation [7, 20], while for $\mu_{\mathrm{B}}=0$ it is identical in form to Ref. [21, Eq. (1)].

Remark 9. The $\mu_{\mathrm{B}} \geq 0$ cases of (47) admit, with $u(x, t)=f(\xi)$, the same TWSs as the corresponding cases of (15); however, under (47) the wave speed is given by $\lambda=\epsilon \beta / 2$; again, see Ref. [21].

Remark 10. It should be noted that (44) can readily be recast to exhibit a nonlinearity of the "RSGC type". As such, the resulting PDE would, on setting $n=1$, reduce to the RSGC special case of Ref. [12, Eq. (19)] corresponding to a non-thermally conducting Newtonian fluid; see also Ref. [22] and those therein.

\section{Discussion}

While the forgoing analysis has reveled a number of new findings regarding weakly-nonlinear acoustic waves in this class of power-law fluids, it is the connection between the special case $n=2$, which we observe does not appear to correspond to a particular fluid, polymer solution, etc., and the finite-scale version of the compressible Navier-Stokes equations (or FSNS for short) that is, in our view, the most interesting of all.

Referring the reader to Ref. [5, 6] for the details of FSNS theory, the connection we have found is the following: The $\mu_{\mathrm{B}}=0$ and $\mu_{\mathrm{B}}>0$ sub-cases of the $n=2$ case of (15) yield TWSs that are identical in form to those admitted by the inviscid and viscous versions, respectively, of the FSNS formulation examined in Ref. [6]; in particular, the quantities u, y, $\epsilon^{-1}$, and $(\gamma+1)$ that appear in Ref. [6, Eq. (24)] correspond6, respectively, to $f, \xi / \sqrt{\alpha}, \sqrt{4 \alpha}$, and $\varkappa^{\bullet}$ in $\left.{ }^{35}\right)_{3}$.

Evidently, the averaging process used to obtain the FSNS from the special case of the compressible (1D) NavierStokes' equations corresponding to $\mu=\eta \varrho$, where the constant of proportionality $\eta$ carries (SI) units $\mathrm{of}^{2} / \mathrm{sec}$, and the bulk viscosity neglected, gives rise to an effective viscosity function that is identical in form to the case $\mu_{\mathrm{B}}>0$, $n=2$ of the present study, but with $\eta$ playing the role of $\nu_{\mathrm{B}}$ (see Remark 3); i.e., as alluded to above, the inviscid limit of Ref. [6, Eq. (24)] corresponds to the case $\mu_{\mathrm{B}}=0, n=2$ (see sect. 3). Also of interest is the fact that $L$, the averaging length parameter in Ref. [6], corresponds to the quantity $4(k v / L) \sqrt{2 / 3}($ with $n=2)$ here.

Given the above, it not surprising that the present study also has a close connection to the work carried out in Ref. [4], wherein the notion of "artificial viscosity" was first introduced in the context of gas dynamics. To see this, one need only compare the $\mu_{\mathrm{B}}=0, n=2$ special case of (3) with Ref. [4, Eq. (3) and (8)]; see also Remark 6.

And lastly, it should be noted that, in addition to compact kinks, both semi-compact front-type, as well as compact pulse-type, TWSs have also been predicted in various types of continuous media; see, e.g., Refs. [19, 23], respectively, and those therein.

\footnotetext{
${ }^{5}$ See, e.g., Crighton's [20, p. 16] treatment of the thermoviscous version of 16 .

${ }^{6}$ This means that the results presented in sect. 4.3 also apply to Ref. [6, Eq. (24)] and/or its solution.
} 


\section{Acknowledgements}

P.M.J. is pleased to acknowledge a series helpful discussions with Dr. Len G. Margolin. P.M.J. was supported by ONR funding.

\section{References}

[1] W.R. Schowalter, Mechanics of non-Newtonian Fluids, Pergamon Press, London, 1978.

[2] F.M. White, Viscous Fluid Flow, 2nd ed., McGraw-Hill, New York, 1991.

[3] B. Straughan, A novel type of wave behaviour in a compressible inviscid dipolar fluid and stability characteristics of generalized fluids, Ann. Mat. Pura Appl. 126 (1980) 187-207.

[4] J. von Neumann, R.D. Richtmyer, A method for the numerical calculation of hydrodynamic shocks, J. Appl. Phys. 21 (1950) $232-237$.

[5] L.G. Margolin, Finite-scale equations for compressible fluid flow, Phil. Trans. R. Soc. A 367 (2009) $2861-2871$.

[6] L.G. Margolin, D.E. Vaughan, Traveling wave solutions for finite scale equations, Mech. Res. Commun. (2012), doi:10.1016/j.mechrescom.2012.07.003 (to appear).

[7] D.G. Crighton, Basic theoretical nonlinear acoustics, in: D. Sette (Ed.), Frontiers in Physical Acoustics, North-Holland, Amsterdam, 1986, pp. $1-52$.

[8] S. Makarov, M. Ochmann, Nonlinear and thermoviscous phenomena in acoustics, Part I, Acustica-Acta Acustica 82 (1996) $579-606$.

[9] R.T. Beyer, The parameter B/A, in: M.F. Hamilton, D.T. Blackstock (Eds.), Nonlinear Acoustics, Academic Press, San Diego, CA, 1997, pp. 25-39.

[10] P.A. Thompson, Compressible-Fluid Dynamics, McGraw-Hill, New York, 1972.

[11] V.P. Kuznetsov, Equations of nonlinear acoustics, Sov. Phys.-Acoust. 16 (1971) 467-470.

[12] P.M. Jordan et al., On the propagation of nonlinear acoustic waves in viscous and thermoviscous fluids, Eur. J. Mech. B/Fluids 34 (2012) $56-63$.

[13] W.D. Hayes, Gasdynamic Discontinuities, Princeton University Press, Princeton, NJ, 1960.

[14] J. Angulo, Nonlinear dispersive equations: existence and stability of solitary and periodic travelling wave solutions, in: Mathematical Surveys and Monographs, vol. 156, American Mathematical Society, Providence, RI, 2009.

[15] G. Saccomandi, Elastic rods, Weierstrass theory and special travelling waves solutions with compact support, Int. J. non-Linear Mech. 39 (2004) 331-339.

[16] W. Kaplan, Ordinary Differential Equations, Addision-Wesley, Palo Alto, CA, 1958.

[17] B.D. Coleman, M.E. Gurtin, Growth and decay of discontinuities in fluids with internal state variables, Phys. Fluids 10 (1967) $1454-1458$.

[18] M. Destrade, P.M. Jordan, G. Saccomandi, Compact travelling waves in viscoelastic solids, Europhys. Lett. (EPL) 87 (2009) 48001.

[19] I.C. Christov, P.M. Jordan, Shock and traveling wave phenomena on an externally damped, non-linear string, Int. J. non-Linear Mech. 44 (2009) 511-519.

[20] D.G. Crighton, Model equations of nonlinear acoustics, Ann. Rev. Fluid Mech. 11 (1979) 11-33.

[21] D. Wei, H. Borden, Traveling wave solutions of Burgers' equation for power law non-Newtonian flows, Appl. Math. E-Notes 11 (2011) $133-138$.

[22] A.R. Rassmusen et al., Interacting wave fronts and rarefaction waves in a second order model of nonlinear thermoviscous fluids: interacting fronts and rarefaction waves, Acta Appl. Math. 115 (2011) 43-61.

[23] P.M. Jordan, G. Saccomandi, Compact acoustic travelling waves in a class of fluids with nonlinear material dispersion, Proc. R. Soc. A (2012), doi: 10.1098/rspa.2012.0321 (to appear). 